\title{
A New Combined Surface and Volume Registration
}

\author{
Natasha Leporée ${ }^{1,2 \star}$, Anand A. Joshi ${ }^{1 \star}$, Richard M. Leahy ${ }^{3}$, Caroline Brun ${ }^{1}$ Yi-Yu Chou $^{1}$, \\ Xavier Pennec ${ }^{4}$, Agatha D. Lee ${ }^{1}$, Marina Barysheva ${ }^{1}$, Greig I. de Zubicaray ${ }^{5}$, Margaret J. \\ Wright $^{6}$, Katie L. McMahon ${ }^{5}$, Arthur W. Toga ${ }^{1}$, Paul M. Thompson ${ }^{1}$ \\ ${ }^{1}$ Laboratory of Neuro Imaging, UCLA School of Medicine, Los Angeles, CA, USA \\ ${ }^{2}$ Department of Radiology, University of Southern California, Children's Hospital Los \\ Angeles, CA, USA \\ ${ }^{3}$ Department of Engineering, University of Southern California, Los Angeles, CA, USA \\ 4 Asclepios Research Project, INRIA Sophia, Sophia-Antipolis Cedex, France \\ ${ }^{5}$ Centre for Magnetic Resonance, University of Queensland, Brisbane, Australia \\ ${ }^{6}$ Genetic Epidemiology Lab, Queensland Institute of Medical Research, Brisbane, Australia
}

\begin{abstract}
3D registration of brain MRI data is vital for many medical imaging applications. However, purely intensitybased approaches for inter-subject matching of brain structure are generally inaccurate in cortical regions, due to the highly complex network of sulci and gyri, which vary widely across subjects. Here we combine a surfacebased cortical registration with a 3D fluid one for the first time, enabling precise matching of cortical folds, but allowing large deformations in the enclosed brain volume, which guarantee diffeomorphisms. This greatly improves the matching of anatomy in cortical areas. The cortices are segmented and registered with the software Freesurfer. The deformation field is initially extended to the full 3D brain volume using a 3D harmonic mapping that preserves the matching between cortical surfaces. Finally, these deformation fields are used to initialize a 3D Riemannian fluid registration algorithm, that improves the alignment of subcortical brain regions. We validate this method on an MRI dataset from 92 healthy adult twins. Results are compared to those based on volumetric registration without surface constraints; the resulting mean templates resolve consistent anatomical features both subcortically and at the cortex, suggesting that the approach is well-suited for cross-subject integration of functional and anatomic data.
\end{abstract}

Keywords: brain, image analysis, Magnetic Resonance Imaging, image registration

\section{INTRODUCTION}

Registration of brain MRI scans is a key step in many medical imaging studies, for multimodality integration, computational anatomy studies, and longitudinal tracking of disease. In non-linear image registration, a template image $T$ is transformed into a study image $S$, by aligning the geometrical features of the images. The anatomical correspondences between images can be measured and optimized in two ways, either based on intensity-derived information at each voxel such as the squared difference in intensities or mutual information between the images, or by using anatomical landmarks, which may include 3D parametric curves and surfaces extracted from the images. These feature correspondences can be used to drive the registration. The image transformation is also typically constrained via a regularizer that is chosen to ensure that the transformation is spatially smooth, invertible, and so that it satisfies other desirable properties such as inverse-consistency or transitivity. ${ }^{15}$

Registration algorithms for brain MR images may also be divided into two major categories based on whether they attempt to register the entire 3D brain volume, or are restricted to the cortical surfaces. Volume-based algorithms perform quite well for subcortical structures, but have difficulty in matching cortical surfaces, due to the high complexity and variability in cortical patterns. Cortical registration is therefore used when the subsequent analysis focuses more specifically on the cortex, e.g. for population-based studies of cortical thickness. In this case, the registration is restricted to the cortical surface, and does not take into account the rest of the brain volume.

$\star$ : equal contribution 
Cortical registrations are usually performed by first transforming the cortices to more regular surfaces such as the plane or the sphere, though some recent algorithms have been developed that directly register the cortices using 3D harmonic maps. ${ }^{25}$ Typically the surface-based registrations either use sulci or gyri as anchors to drive the transformation, ${ }^{30}$ or alternatively, make use of quantities defined over the whole brain such as the curvature $^{7}$ or the local conformal factor. ${ }^{31}$ To compute the flows in surface coordinates, specialized approaches using covariant PDEs, currents, or holomorphic 1-forms have been developed. ${ }^{9}$

There are many ways to perform the volume-based registrations. In this work, we focus on regularizers that assign continuum mechanical laws to the deforming image medium. The most popular model for these regularizers is the elastic one. Here a similarity function is selected, and a force is derived from it and applied to the system, while the elastic forces attempt to restore the original shape of the medium. Elastic registration is a good choice for studies where small deformations are expected, such as longitudinal studies where the same subject is imaged at two time points, and small changes are expected. However, in cases where large differences exist between the two images, the deformation can induce tearing or shearing of the elastic medium. In this case, other methods are needed. Among these, the fluid registration method was first introduced in. ${ }^{6}$ In this case, the image is treated as a viscous fluid that obeys a linearized version of the Navier-Stokes equation. The velocity $\vec{v}$ of the fluid is the time derivative of the deformation field $\vec{v}(\vec{r}, t)=\frac{d \vec{u}(\vec{r}, t)}{d t}$. The driving force for the flow is again generated by a similarity function. The fluid paradigm allows large deformations without shearing or tearing of the image.

A new elastic regularizer was recently introduced in. ${ }^{21}$ In this case, the regularization is based on the deformation tensors $\Sigma=(\nabla \overrightarrow{(u)}+I)^{T}(\nabla \vec{u}+I)$, with $I$ the identity matrix. The $\Sigma$ 's characterize the voxelwise changes in shape and volume caused by the registration. As the $\Sigma$ 's are positive-definite symmetric matrices, the computations are performed in the Log-Euclidean framework, ${ }^{1}$ which allows analytical computations on the manifold of $\Sigma$ 's. A fluid version of this method was developped in, ${ }^{5}$ where the rate of change of $\Sigma$ was regularized instead of $\Sigma$.

In this work, we combined surface and volume based algorithms, to overcome serious weaknesses of each component code that severely limit their usefulness (one is restricted to the surface, the other cannot handle the cortex accurately). Although combination of the two is likely to be much more useful, as it allows good matching everywhere, no hybrid registration approach is yet widely used, ${ }^{27} .{ }^{29}$

There are several ways to achieve a good registration over the whole brain volume, including the cortex. The simplest one, which we investigate here, is to use a cortical surface registration to obtain an initialization for the volume registration. This allows a slightly simpler implementation than one that allows the surface deformation to evolve while the volumes are matched. A similar method was designed in ${ }^{13}$ where a new algorithm was introduced that performs both surface and volume registrations ( $\left(\mathrm{see}^{\mathrm{also}} \mathrm{s}^{22}\right.$ ). A landmark-based registration is first performed on the cortical surfaces, by constraining the matching between sulci. The correspondence was then extended to the whole brain using a constrained 3D harmonic mapping. The third step consists of refining the volume alignment via elastic registration.

Here we improve on the method from ${ }^{13}$ in two ways. The algorithm in ${ }^{13}$ gives excellent matching in cortical areas. However, an elastic registration is used to align the subcortical structures. This method gives poor results when large deformations are required, and is not provably diffeomorphic, i.e., there is no mathematical guarantee that the interior mapping is invertible with a smooth inverse. This latter property is vital for tensorbased morphometry, a powerful approach for large-scale analysis of regional brain volumes and shape using statistics of the local deformation, ${ }^{17} \cdot{ }^{11}$

Here we combine a cortical registration with a fluid one for the first time, enabling precise matching of cortical folds, but allowing large deformations in the enclosed brain volume, which guarantee diffeomorphisms. For this, we will use the Riemannian fluid algorithm developed in. ${ }^{5}$ The Riemannian fluid is regularized to minimize the distortion at each voxel from the registration. Furthermore, the surface matching algorithm designed in ${ }^{13}$ uses sulci as landmarks to anchor the cortical registration. This method is prohibitive for large datasets, as the sulci need to be traced by hand. Here instead we use the Freesurfer software ${ }^{7}$ (which makes use of a curvature-based registration algorithm) to align the cortical surfaces. As a result, the entire registration process is automated. 
We validate our new combined algorithm using a large dataset of brain MRI images from 92 healthy adult subjects, divided into 23 pairs of monozygotic twins (MZ) and 23 same-sex pairs of dizygotic twins (DZ) from which we also obtain whole brain correlation statistics assessing the anatomical similarity between twins.

\section{METHOD}

\subsection{Surface-Volume Registration}

Our method aims to produce a one-to-one mapping between two brain volumes such that the subcortical structures and the cortical surfaces are aligned. Formally, let $M$ and $N$ represent the subject and target brain white matter volumes and let $S$ and $T$ denote the volumetric images such that $S(x), x \in M$, is the image intensity value at point $x$ in the subjects volume $M$ and $T(x), x \in N$, is the image intensity value at point $x$ in the target volume $N$. The cortical surface $\partial M$ of the subject $M$ is the boundary of the subject white matter volume $M$ and the cortical surface $\partial N$ of the subject $N$ is the boundary of the target brain's white matter volume $N$. There are two mains steps in our algorithm. The first step extends the surface-based registration to the enclosed $3 \mathrm{D}$ volume by using a 3D harmonic mapping based method ${ }^{13}$ and the second step performs the alignment of subcortical volumes by Riemannian fluid ${ }^{4,5}$ intensity registration.

Our method consists of:

1. Surface matching: A map is computed between $\partial M$ and $\partial N$, the cortical surfaces of the subject and target brains, respectively. The registrations are done from each of the subjects cortices to that of the target brain.

2. Extrapolation of the surface map to the entire enclosed cerebral volume, such that the cortical surfaces remain aligned: This is done by computing a 3D harmonic map $u$ from $M$ to $N$ while prescribing the deformation field defined on the cortices as a boundary condition, as in. ${ }^{13}$ The map is computed as follows (see ${ }^{13}$ for more details). First, $M$ and $N$ are both mapped to the surface of the unit sphere using Freesurfer. ${ }^{7}$

Let $g_{\alpha \beta}$ and $h_{\alpha \beta}$ denote the metrics associated with the mapping of $M$ and $N$ to the unit sphere, respectively. The harmonic map $u: M \rightarrow N$ can be computed by minimizing the mapping energy:

$$
E(u)=\frac{1}{2} \int_{M} \sum_{i, j=1}^{3} \sum_{\alpha, \beta=1}^{3} h_{\alpha \beta}(u(x)) \frac{\partial u^{\alpha}(x)}{\partial x^{i}} \frac{\partial u^{\beta}(x)}{\partial x^{j}} d \mu_{g} .
$$

As a result of these two steps, we get a diffeomorphism between the two brain volumetric manifolds $M$ and $N$. The map aligns the folding patterns of the sulci but does not align the subcortical structures because the intensity values are not used in this step. This map is refined further in the next step using Riemannian fluid-based intensity registration. ${ }^{5}$ A brief description of the method can be found in the following section.

\subsection{Volume Registration}

We register the outputs of the previous stage further for subcortical alignment using the Riemannian fluid registration algorithm. ${ }^{5}$

Fluid registration proceeds in a series of time steps $d t$. At each time $t$ and voxel $\vec{x}$, the velocity $\vec{v}$ is found from:

$$
\frac{d \vec{v}(\vec{x}, t)}{d t}=\nabla \operatorname{Cos} t+\nabla \operatorname{Reg}(\vec{v}, t)-\vec{v}
$$

Cost is the similarity cost function and Reg is the regularizer. Once the registration is completed, $\vec{v}$ is integrated over time to obtain the final displacement field $\vec{u}$.

In particular, for the Riemannian fluid registration, ${ }^{5}$ the regularization is done on the matrix logarithm of the rate of strain $\Sigma_{v}=(\nabla \vec{v}+I)^{T}(\nabla \vec{v}+I)$ :

$$
\operatorname{Reg}_{\text {Riem }}(\vec{v}, t)=\int \frac{\mu}{4} \operatorname{Tr}\left(\log \left(\Sigma_{v}^{2}\right)\right)+\frac{\lambda}{8} \operatorname{Tr}\left(\log \left(\Sigma_{v}\right)\right)^{2} .
$$


Here $\mu$ and $\lambda$ are fluid parameters to be selected by the user (analogous to the Lamé coefficients). Reg Riem is the fluid equivalent of the Riemannian elastic regularizer from ${ }^{21}$ the fluid case regularizes the rate of strain $\Sigma_{v}$ rather than the deformation tensors $\Sigma_{u}=(\nabla \vec{u}+I)^{T}(\nabla \vec{u}+I)$ in Eq. 2. The tensor field $\Sigma$ characterizes the local shape and volume deformation. These tensors live on the curved manifold of positive-definite, symmetric matrices. Taking the matrix logarithm of $\Sigma$ transports it to the tangent plane at the origin of the manifold, which is a flat space (vector space) in which simple Euclidean computations can be performed. ${ }^{1}$

We chose an intensity-based cost function that penalizes the squared difference in intensity between the deforming template $T$ and the study $S$ :

$$
\operatorname{Cost}(\vec{x}, \vec{u}(\vec{x}, t))=(T(\vec{x}-\vec{u}(\vec{x}, t))-S(\vec{x}))^{2}
$$

We note that intensity matching terms based on mutual information, correlation, or other metrics could be used instead, but we do not cover these here. See ${ }^{4}$ for details of the implementation.

As a result of this step, the diffeomorphic map generated in the previous step is refined to align the subcortical structures.

\subsection{Non-linear Registration}

We performed all 3 of the steps described in section 2.1 between each image in the dataset and a template, which was chosen randomly as one of the subjects images. Alternatively, as in our past work, a geometrically-centered mean template could be generated and used instead. ${ }^{16}$

For each subject, we obtained a displacement field $\overrightarrow{u_{1}}$ and a registered image from the surface matching and the subsequent harmonic mapping. The displacement field $\overrightarrow{u_{1}}$ was used as input for the Riemannian fluid algorithm described in sec 2.2 . The volume registration yielded a vector field $\overrightarrow{u_{2}}$. We used the vector fields from the surface registration as input instead of the registered images. A diffeomorphic map between the surfaces (such as those from Freesurfer and the harmonic extension to the whole brain volume) is provided as an initial input for the fluid registration; this also ensures that the final results will also be diffeomorphic, as the composition of two diffeomorphic maps. ${ }^{6}$ In our implementation, the final displacements were computed from the common template to each image in the data set, while the registered image was the result of transforming each subject into the template, thus allowing all comparisons to be made in the common space.

\section{RESULTS}

\subsection{Data}

We analyzed 3D structural brain MRI scans of 23 monozygotic (MZ) and 23 dizygotic (DZ) same-sex twin pairs (age range: $22-25$ years), as well as one of an identically scanned healthy subject used as the common target for the fluid registration. Our images were collected using a 4 Tesla Bruker Medspec whole body scanner (Bruker Medical, Ettingen, Germany) at the Center for Magnetic Resonance (University of Queensland, Australia). Three-dimensional $T 1$-weighted images were acquired with a magnetization prepared rapid gradient echo $(M P$ $R A G E)$ sequence to resolve anatomy at high resolution. Acquisition parameters were: inversion time $(T I)$ $/$ repetition time $(T R) /$ echo time $(T E)=1500 / 2500 / 3.83 \mathrm{msec}$; flip angle $=15^{\circ}$; slice thickness $=0.9 \mathrm{~mm}$ with a $256 \times 256 \times 256$ acquisition matrix.

Extracerebral (non-brain) tissues were manually deleted from the MRI images using the 3D interactive program Display (Montreal Neurological Institute, McGill University, Canada). All scans were then aligned to the ICBM53 template using 9-parameter registration (i.e., translational and rotational alignment, allowing scaling in 3 independent directions) found in the FMRIB's Linear Image Registration Toolbox, FLIRT. ${ }^{12}$ 


\subsection{Twin Statistics}

For each subject, at each voxel, the displacement $u$ was computed, as well as the deformation matrix $\Sigma$ and the determinant of the Jacobian matrix $J=(I d+\nabla u) \cdot \operatorname{det}(J)$ measures local volume expansions, where $\operatorname{det}(J)>1$, and shrinkage when $\operatorname{det}(J)<1$. In the full set of MZ and DZ twins, the resemblance between twin pairs at each voxel can be computed from $\operatorname{det}(J)$ to assess the Intraclass Correlation: ${ }^{23}$

$I C C=\frac{\sigma_{b}^{2}}{\left(\sigma_{b}^{2}+\sigma_{w}^{2}\right)}$, where $\sigma_{b}^{2}$ and $\sigma_{w}^{2}$ are the pooled variance between pairs and within pairs, respectively. We did not want to assume that the data on volumetric differences (here $\operatorname{det}(J)$ ) were normally distributed, so we computed $p$ values at each voxel with a voxelwise permutation test (5000 permutations), to establish a null distribution for the ICC statistics at each voxel (the null hypothesis was $I C C=0$ (no correlation)).

\subsection{Details of the Numerical Implementation}

We used Freesurfer for segmentation of the brains and extraction of the cortical surface meshes. The surface registration step in Section 2.1 was performed by Freesurfer by using a spherical parameterization of the cortex and a metric representing cortical 'depth' ${ }^{7}$ that is a function of the surface curvature. The extension of the surface registration to the full volume, described in Section 2.1, and the Riemannian fluid algorithm, in Section 2.2, were implemented in MATLAB. To test our method, we selected one brain from the database as a target brain volume and registered the rest of the brains to it. On an Intel Pentium IV processor the registration of each subject to a target image takes approximately 1 hour.

\subsection{Registration Results}

Figure 1 shows a comparison of the registration from the fluid code alone, without cortical surface constraints, compared to the combined code, which also matches the cortex. The fluid registration alone gives poor results in some areas, e.g., most of the cortex. This is a serious problem for all fluid algorithms, hence the initialization with the cortical registration used here. Initializing with the displacements from the cortical registration noticeably improves the cortical results. Fig. 1 also shows that the subcortical structures are now also better aligned; this may be due to deep cortical surfaces passing near the them, especially at the corpus callosum.

The top row in Figure 2 shows the results of averaging the intensities of all 92 registered images. For the results using the proposed hybrid algorithm, features at the cortical boundary and the ventricles are still clearly defined after averaging. This is in marked contrast with those from the Riemannian registration alone, where many key anatomical features are blurred away by the averaging. We can see that alignment of the surface gives sharper images near the surface boundary. We also observed less blurring for the subcortical structures such as the ventricles. The second row show the variance in intensity at each voxel for the images in our dataset. The variance is much lower for the combined algorithm. Again this is true even in subcortical areas, except within the ventricles.

\section{DISCUSSION}

We investigated a new combined algorithm for the registration of brain MR images. Our algorithm was tested on a large data set of 92 healthy adult twins, and showed significant improvement over using fluid registration alone, both for subcortical areas and in the vicinity of the cortex. Intraclass correlation statistics were significantly improved over using fluid registration alone. The premise of using twin data to help validate registration is that any errors in registration will tend to be uncorrelated between twins, and will tend to deplete the natural correlation in brain anatomy that is due to genetic factors.

There are several ways to achieve a good registration over the whole brain volume, including the cortical surface. The simplest one, which we investigated here, is to use a cortical surface registration to initialize a $3 \mathrm{D}$ registration. In that case, as we showed here, the matching converges to better local minimum compared to the one found from the fluid registration alone. However, as the cortex is allowed to move during the fluid registration, it can potentially reduce the accuracy of the matching found from the cortical matching algorithm. Thus, as an alternative, one could choose instead to re-introduce the cost function from the first cortical matching algorithm into the volume registration, either as a hard constraint or with a Lagrange multiplier. As the matching was already quite good with the first method, this second method was not pursued here, though it would be an 


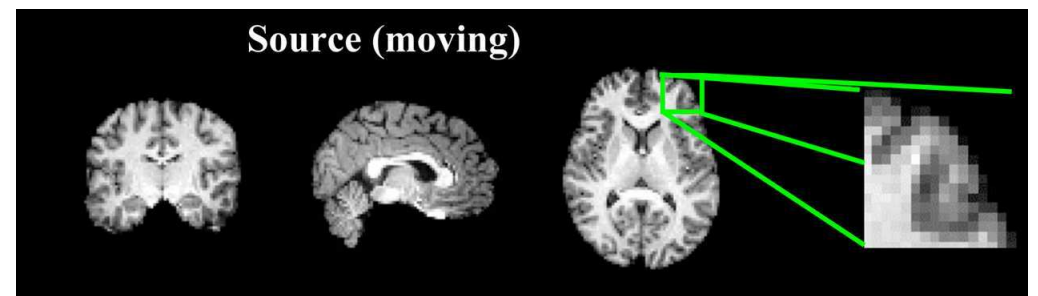

Target (fixed)

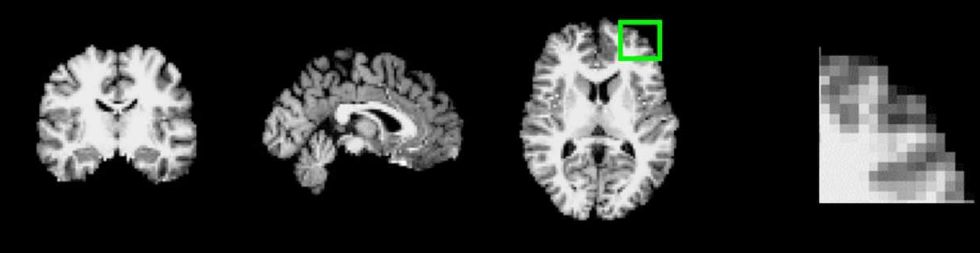

Volume registration
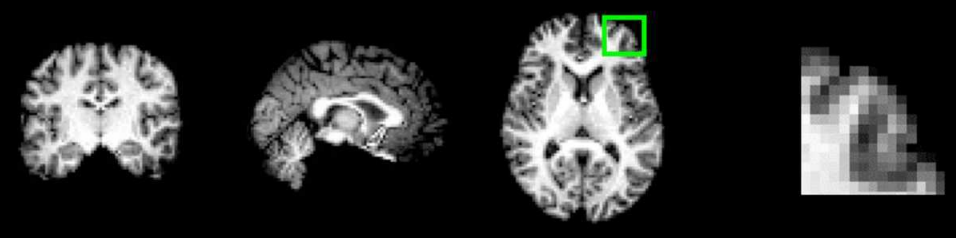

Volume and surface registration
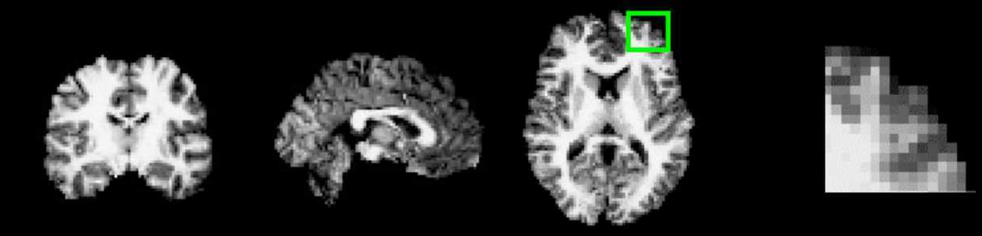

Figure 1. Comparison of the results of the combined algorithm to those of the 3D Riemannian algorithm with no surface constraints. Registration results are shown mapping one subject to a template in coronal (left column), sagittal (middle column) and horizontal (right column) slices. The top row shows the moving image and the second row shows the fixed target image. The last two rows show the results of the registration for the volumetric registrations without surface constraints (third row) and with constraints (fourth row); the fourth row shows accurate cortical and subcortical registration. Results are more accurate for the combined code both on the cortex (see boxed area in figure) and for subcortical structures. 

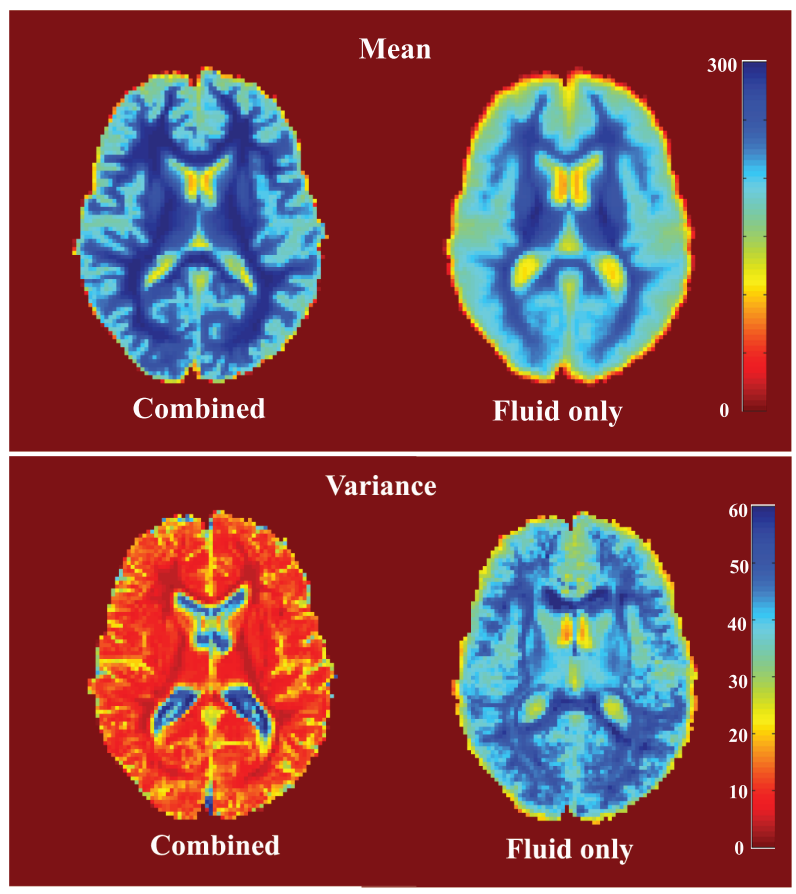

Figure 2. The top row shows the result of averaging the intensities for all 92 registered images, with the colorbar showing the mean intensity values. Anatomical features near the cortex are much sharper when the combined code is used. The bottom row shows the voxelwise variance in intensity in these same registered images, with the variance values shown according to the colorbar on the right. The top and bottom left images are for our combined algorithm, while the ones on the right are for the Riemannian algorithm alone. The intensity variance in the registered images is much lower with the combined algorithm, which suggests that misalignment of cortical anatomy is reduced.

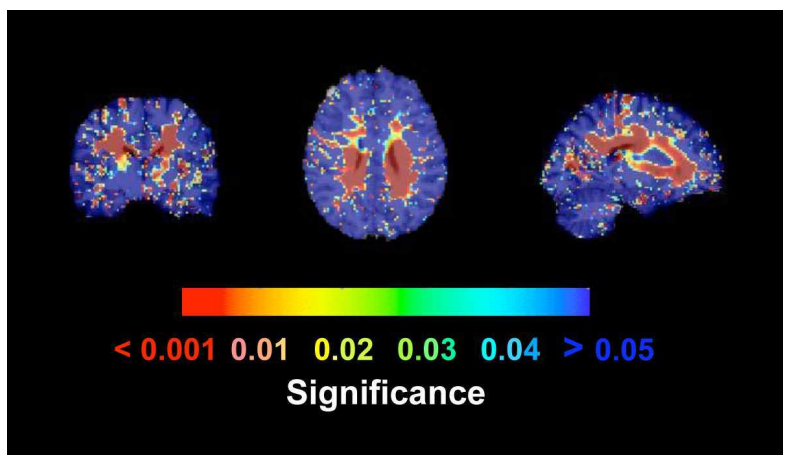

Figure 3. Maps of p-values for the Intraclass correlation for the monozygotic twins. Sulcal patterns are known vary naturally even among monozygotic twins, but subcortical anatomy is under strong genetic control; areas of highly significant correlation include the anterior cingulate and paralimbic regions. 
interesting extension of this work. Finally, in cases where both the above methods fail, a third option would be to force the displacements from the Riemannian fluid algorithm to match those found from the cortical mapping.

As a next step, a large-scale group validation is needed to compare the performance of our algorithm to other state-of-art registration algorithms. In the near future, we plan to use a validation such as the one in $^{14}$ to further validate our method. Because our approach produces diffeomorphic mappings while enforcing cortical constraints, it is likely to improve results in tensor-based morphometry studies, which are now being conducted on a large scale to assess the effects of disease, medication, and specific genetic polymorphisms on brain structure.

\section{Acknowledgments}

This work was supported by NIH grant R01 HD050735 and the National Health and Medical Research Council, Australia grant 496682.

\section{REFERENCES}

1. Arsigny V, Fillard P, Pennec X, Ayache N, Log-Euclidean metrics for fast and simple calculus on diffusion tensors, Mag Res Med 56, (2006) 411-421.

2. Ashburner J, A fast diffeomorphic image registration algorithm, Neuroimage 38, (2007), 95-113.

3. Brun C, Leporé N, Pennec X, Chou YY, Lopez OL, Aizenstein OJ, Becker JT, Toga AW, Thompson PM, Comparison of Standard and Riemannian fluid registration for Tensor-Based Morphometry in HIV/AIDS, Workshop on Statistical Registration: Pair-wise and Group-wise alignment and atlas formation, MICCAI, Brisbane, Australia (2007).

4. Brun C, Leporé N, Pennec X, Chou YY, Lee AD, de Zubicaray G, McMahon K, Wright M, Barysheva M, Toga AW, Thompson PM, A new registration method based on Log-Euclidean tensor metrics and its application to genetic studies, Proceedings of the 5th IEEE International Symposium on Biomedical Imaging (ISBI), Paris, France, May 14-17, (2008).

5. Brun C, Leporé N, Pennec X, Chou YY, McMahon K, de Zubicaray GI, Meredith M, Wright MJ, Lee AD, Barysheva M, Toga AW, Thompson PM, A Tensor-Based Morphometry Study of Genetic Influences on Brain Structure using a New Fluid Registration Method, Proceedings, 11th International Conference on Medical Image Computing and Computer Assisted Intervention (MICCAI), New York, NY, USA, Sept 6 10 (2008).

6. Christensen EG, Rabbitt RD, Miller MI, Deformable templates using large deformation kinematics, IEEE Trans Imag Process 5, (1996) 1435-1447.

7. Dale AM, Fischl B, Sereno MI, Cortical surface-based analysis. I. Segmentation and surface reconstruction, Neuroimage 9, (1999) 179-94.

8. Davatzikos C, Vaillant M, Resnick S, Prince J L, Letovsky S, Bryan RN, A computerized approach for morphological analysis of the corpus callosum, J Comput Assist Tomog 20, (1996) 88-97.

9. Durrleman S, Pennec X, Trouvé A, Thompson PM, Ayache N, Inferring Brain Variability from Diffeomorphic Deformations of Currents: An Integrative Approach, Med Imag Anal 12, (2008) 626-37.

10. Gramkow C, Registration of $2 D$ and $3 D$ medical images, Master's thesis, Danish Technical University, Copenhagen, Denmark (1996).

11. Hua X, Leow AD, Parikshak N, Lee S, Chiang MC, Toga AW, Jack CR, Weiner MW, Thompson PM, Tensor-Based Morphometry as a Neuroimaging Biomarker for Alzheimer's Disease: An MRI Study of 676 AD, MCI, and Normal Subjects, Neuroimage 43, (2008) 458-469.

12. Jenkinson M, Bannister PR, Brady JM, Smith SM, Improvised optimization for the robust and accurate linear registration and motion correction of brain images, Neuroimage, 17, (2002) 825-841

13. Joshi AA, Shattuck DW, Thompson PM, Leahy RM, Surface-Constrained Volumetric Brain Registration using harmonic mappings, IEEE-Trans Med Imag 26, (2007) 1657-1669.

14. Klein A, Andersson J, Ardekani BA, Ashburner J, Avants B, Chiang MC, Christensen GE, Collins DL, Hellier P, Hyun PSJ, Lepage C, Pennec X, Rueckert D, Thompson PM, Vercauteren T, Woods RP, Mann JJ, Parsey RV, Evaluation of 14 nonlinear Deformation Algorithms Applied to Human Brain MRI Registration, Neuroimage 46, 786-802 (2009). 
15. Leow AD, Chiang MC, Yanovsky I, Lee AD, Lu A, Klunder AD, Becker JT, Davis SW, Toga AW, Thompson PM, Statistical properties of Jacobian maps and inverse-consistent deformations in non-linear image registration, IEEE Trans Med Imag 26, (2007) 822-832.

16. Leporé N, Brun C, Pennec X, Chou YY, Lopez OL, Aizenstein HJ, Becker JT, Toga AW, Thompson PM, Mean template for Tensor-Based Morphometry using deformation tensors, Proceedings, 10th International Conference on Medical Image Computing and Computer Assisted Intervention (MICCAI), Brisbane, Australia, Oct 29 - Nov 2 (2007).

17. Leporé N, Chou YY, Lopez OL, Aizenstein HJ, Becker JT, Toga AW, Thompson PM, Generalized TensorBased Morphometry of HIV/AIDS using multivariate statistics on deformation tensors, IEEE-Trans Med Imag 27, (2008) 129-141.

18. Leporé N, Chou YY, Lopez OL, Aizenstein HJ, Becker JT, Toga AW, Thompson PM, Fast 3D Fluid Registration of Brain Magnetic Resonance Images, Proceedings, SPIE conference on Physiology, Function and Structure from Medical Images, San Diego, CA, USA, February 16-21 (2008).

19. Leporé N, Brun C, Chou YY, Lee AD, Barysheva M, Pennec X, McMahon K, Meredith M, de Zubicaray GI, Wright M, Toga AW, Thompson PM, Best individual template selection from deformation tensor minimization, Proceedings, International Workshop on Biomedical Imaging (ISBI), Paris, France, April 14-17 (2008).

20. Nichols TE, Holmes AP, Non parametric permutation tests for functional neuroimaging: a primer with examples, Hum Brain Map 15, (2001) 1-25.

21. Pennec X, Stefanescu R, Arsigny V, Fillard P, Ayache N, Riemannian elasticity: A statistical regularization framework for non-linear registration, Proceedings, Medical Image Computing and Computer-Assisted Intervention (MICCAI), Palm Springs, CA, USA, (2005) 943-950.

22. Postelnicu G, Zollei L, Fischl B, Combined volumetric and surface registration, IEEE Trans Med Imag 28, (2008) 508-522.

23. Scout PE, Fleiss JL, Intraclass correlations: Uses in assessing rater reliability, Psychol Bull 86, (1979) 420-428.

24. Shattuck DW and Leahy RM, BrainSuite: an automated cortical surface identification tool, Med Imag Anal 6, (2002) 129-141.

25. Shi Y., Thompson, P.M., Dinov, I., Osher, S., Toga, A.W., Direct cortical mapping via solving partial differential equations on implicit surfaces, Med Imag Anal 11, (2007) 207-223.

26. Storey JD, A Direct Approach to False Discovery Rates, J R Stat Soc. B 64, (2002) 479-498.

27. Thompson PM, Toga AW, A Surface-Based Technique for Warping 3-Dimensional Images of the Brain, IEEE Trans Med Imag 15, (1996) 402-417.

28. Thompson PM, Giedd JN, Woods RP, MacDonald D, Evans AC, Toga AW, Growth Patterns in the Developing Brain Detected By Using Continuum-Mechanical Tensor Maps, Nature 404, (2000) 190-193.

29. Thompson PM, Woods RP, Mega MS, Toga AW, Mathematical/Computational Challenges in Creating Population-Based Brain Atlases, Hum Brain Mapp 9, (2000) 81-92.

30. Thompson PM, Cannon TD, Narr KL, Van Erp T, Poutanen VP, Huttunen M, Lönnqvist J, StandertskjöldNordenstam CG, Kaprio J, Khaledy M, Dail R, Zoumalan CI, Toga AW, Genetic influences on brain structure, Nat Neurosci 4, (2001) 1253-1258.

31. Wang YL, Chiang MC, Thompson PM, Automated Surface Matching using Mutual Information Applied to Riemann Surface Structures, Proceedings, Medical Image Computing and Computer Assisted Interventions (MICCAI), Palm Springs, CA, Oct. 26-29, (2005). 